\title{
Temporal dynamics of generalization and representational distortion
}

\author{
Matthew G. Wisniewski, Barbara A. Church, and Eduardo Mercado III \\ University at Buffalo, State University of New York, Buffalo, New York
}

\begin{abstract}
Individuals generalize differently depending on the extent of their past experiences and what they learn from them. For instance, the peak of generalization can shift from a familiar stimulus to novel ones when the familiar stimulus has been repeatedly discriminated from another, similar stimulus. We examined how the amount of experience students had in discriminating complex sounds impacted their later generalization. As training increased, participants improved at distinguishing sounds, but their tendency to respond to certain novel sounds increased. With additional training, however, this shift dissipated. The results suggest that shifts in generalization may correspond to transitional states of perceptual learning and that learning-related changes in perceptual sensitivities involve more than just incremental increases in feature selectivity.
\end{abstract}

People generalize what they learn about perceived events (Shepard, 1987). Typically, the more perceptually similar new events are to familiar ones, the more likely one is to generalize. For instance, we generalize the identity of objects to views at orientations we have never experienced, but generalization decreases as novel orientations become less similar to familiar views (Ashworth \& Dror, 2000).

Learning experiences can change the way we generalize and, in some instances, can cause novel stimuli to be recognized more often than a trained stimulus (Baron, 1973; Blough, 1975; Dukhayyil \& Lyons, 1973; Galizio, 1980; Gibson, 1969; Guillett et al., 2010; Hearst, 1971; Livesey \& McLaren, 2009; Lynn, Cnaani, \& Papaj, 2005; McLaren \& Mackintosh, 2002; Purtle, 1973; Spence, 1937; Spetch, Cheng, \& Clifford, 2004; Terrace, 1966; Thomas, Mood, Morrison, \& Wiertelak, 1991; Wills \& Mackintosh, 1998; Wisniewski, Church, \& Mercado, 2009). For example, a person might be more accurate at recognizing and naming a caricature of Richard Nixon than a photograph of him. The photograph would be more physically similar to previously experienced Richard Nixon images, but the caricature would likely have exaggerations of features that make his face unique. These exaggerations of learned unique features likely contribute to the rapid recognition and higher identification accuracy for caricatures (Gibson, 1969).

People trained to distinguish faces in the laboratory show similarly distorted sensitivities. Spetch et al. (2004) formed a continuum of faces by morphing a unique face with an average face. People were trained to identify a particular face on the continuum by responding "yes" whenever it was presented and "no" whenever a similar face was shown. Later, when they were shown many faces along this continuum, they gave "yes" responses most often to a novel face that was displaced further along the continuum from the face that they responded "no" to during training. This effect has been seen with several other methods, modalities, and stimulus dimensions. For instance, social stereotypes can result from attributes associated with familiar stimuli being strongly endorsed for novel stimuli that have similar physical characteristics (Queller, Schell, \& Mason, 2006). Also, auditory discrimination training has led to shifts in the identification of frequency-modulated sweeps (chirping sounds) of different repetition rates (Wisniewski et al., 2009) and birdsong notes from a different category than the trained note (Guillett et al., 2010). Such learning-related shifts in generalization are called peak shifts, because the peak of endorsement occurs at a stimulus other than the one that was trained. The size of peak shift depends on the similarity between the exemplars experienced during training, with a certain degree of similarity needed to produce the effect and greater similarity typically leading to larger peak shifts (for a review, see Purtle, 1973).

Several theories explain peak shift by assuming incremental adjustments of weights between static stimulus representations and outputs (Blough, 1975; Guillett et al., 2010; McLaren \& Mackintosh, 2002; Petrov, Dosher, \& Lu, 2005; Saksida, 1999; Wills \& Mackintosh, 1998). ${ }^{1}$ These theories posit that when an event is experienced, it activates multiple representational elements, some of which are shared across different events and some of which are unique to that event. During learning, unique elements become more strongly associated with the output. If a novel event activates these elements to a greater degree than does the trained stimulus, an individual will respond more to the novel event. These incremental theories predict that peak shift should change in parallel with learning. Specifically, if learning increases the weights

M. G.Wisniewski, mgw@buffalo.edu 
of fixed distinctive elements, peak shift should become increasingly evident as a person learns, because learning increases emphasis on those unique elements.

From this perspective, peak shift reflects modifications in processing that facilitate discrimination, are an adaptive means for classifying stimuli, and should develop progressively before reaching a stable end state. In accordance with these ideas, some animal researchers have hypothesized that generalization shifts are an end state of learning important to species survival (Lynn et al., 2005). Even within the uniquely human domain of language, peak shift is seen as an end state in the perceptual learning of phoneme categories (for a review, see Jusczyk \& Luce, 2002) and is thought to contribute to phonological evolution across generations (Martindale, 2006).

Peak shift, however, may not always be an adaptive end state if it results in the endorsement of incorrect categories (Guillett et al., 2010) or stereotyping (Queller et al., 2006). Peak shift may be partially beneficial because it results in the separation of training stimuli, but it also has the adverse consequence of reducing the precision of discrimination abilities with other stimuli. Furthermore, shift may not always be an end state, because the perceptual similarity that modulates peak shift can drastically change over the course of practice and some of those changes may not involve incremental reweighting of connections between representations and outputs. For instance, the neural representations evoked by the presentation of different stimuli can become less similar through spatial separation within the cortex, so that there is less neural overlap between stimuli (Blake, Heiser, Caywood, \& Merzenich, 2006; Blake, Strata, Churchland, \& Merzenich, 2002; Goldstone, 1998; Mercado, 2008; Polley, Steinberg, \& Merzenich, 2006). Learned adjustments of attention can have comparable effects by enhancing the resolution of different dimensions or features in a stimulus (Nosofsky, 1986). Switching the type of representation or processing strategy used to characterize a category can also alter similarity to the category (Johansen \& Palmeri, 2002; Smith \& Minda, 1998). Because neural circuits differ in their capacity to resolve fine details, it has been hypothesized that decreased perceptual similarity can come about by learning to switch the locus of stimulus processing to circuits with the most appropriate perceptual resolution (Ahissar, Nahum, Nelken, \& Hochstein, 2009). These proposed mechanisms assume that representations for experienced events are dynamic over the course of learning and that they may change in nonlinear ways. If the representations or processes become dissimilar enough with practice, there may be a point at which peak shift no longer occurs. These theories predict that peak shift may be a transitional state, rather than the end state of learning.

There have been relatively few investigations into how different amounts of training affect peak shift. In one study, Galizio (1980) found that participants trained for 40 trials showed more shift on an auditory frequency continuum than did participants trained for 10 trials, suggesting an incremental increase in peak shift. In another study, Terrace (1966) trained pigeons on a wavelength discrimination and tested generalization after 15, 30, 45, and 60 training sessions. He found that peak shift was prominent after 15 training sessions but was no longer present after 60 training sessions, showing that peak shift can dissipate with extended training. However, later pigeon experiments did not show this effect. Some pigeons trained for as many as 115 sessions continued to show a peak shift effect (Dukhayyil \& Lyons, 1973; Hearst, 1971). The previous studies are thus equivocal with respect to the stability of peak shift, even though incremental-learning theories have specific predictions about what should happen.

To further investigate the impact of different amounts of discrimination training on shifts in generalization, we trained participants to discriminate frequency-modulated (FM) sweeps of different repetition rates. Groups of participants were trained for different numbers of trials and were then tested using a wide range of stimuli along the rate continuum. To our knowledge, all incremental theories of learning predict that peak shift should become stronger with increased amounts of training. On the other hand, theories assuming nonlinear learning dynamics (because they either allow representations to change or allow changes in processes) predict nonlinear transitions in generalization. We expected to see the latter.

\section{METHOD}

\section{Participants}

One hundred two participants from the University at Buffalo, State University of New York, participated in partial fulfillment of their introductory psychology course requirements. Seventeen participants were randomly assigned to groups trained for 60, 100, 140, 180,220 , or 260 trials. Three participants, one each from the groups trained for 60,140 , and 180 trials, were dropped for excessive "target" responding (over 50\%) in the generalization test. One participant from each of the remaining groups was randomly dropped to create equal sample sizes.

\section{Stimuli and Apparatus}

Stimuli were 1-sec-long sounds consisting of trains of FM tonal sweeps (created with MATLAB 6.5). Individual sweeps increased in frequency from 500 to $4000 \mathrm{~Hz}$ (see Figure 1). All the sounds were the same duration and spanned the same range of frequencies but varied in repetition rate. Subjectively, the stimuli sound like chirps that vary in how fast they are played. The repetition rates used were 4.5, 5.2, 6.0, 6.9, 7.9, 9.1, 10.5, and 12.1 Hz. Earlier work showed that training with these sounds reliably produced peak shift (Wisniewski et al., 2009). Sounds were presented and responses were collected using DMDX experimental software (Forster \& Forster, 2003) on IBM-compatible computers. Participants heard the sounds at conversational volume through Audio-Technica ATH-M40fs headphones and responded using a computer keyboard.

\section{Design}

The design was a $6 \times 8$ mixed factorial with training group as the between-participants and stimulus rank as the within-participants factors. The six levels of training group were 60, 100, 140, 180, 220, and 260 training trials, respectively. The eight levels of the stimulus rank fixed factor were created by rank ordering the repetition rates as follows: $1(4.5 \mathrm{~Hz}), 2(5.2 \mathrm{~Hz}), 3(6.0 \mathrm{~Hz}), 4(6.9 \mathrm{~Hz}), 5(7.9 \mathrm{~Hz})$, $6(9.1 \mathrm{~Hz}), 7(10.5 \mathrm{~Hz})$, and $8(12.1 \mathrm{~Hz})$.

\footnotetext{
Procedure

Participants were instructed to press the right shift key marked "Target" when they heard the target sound and to press the left shift
} 

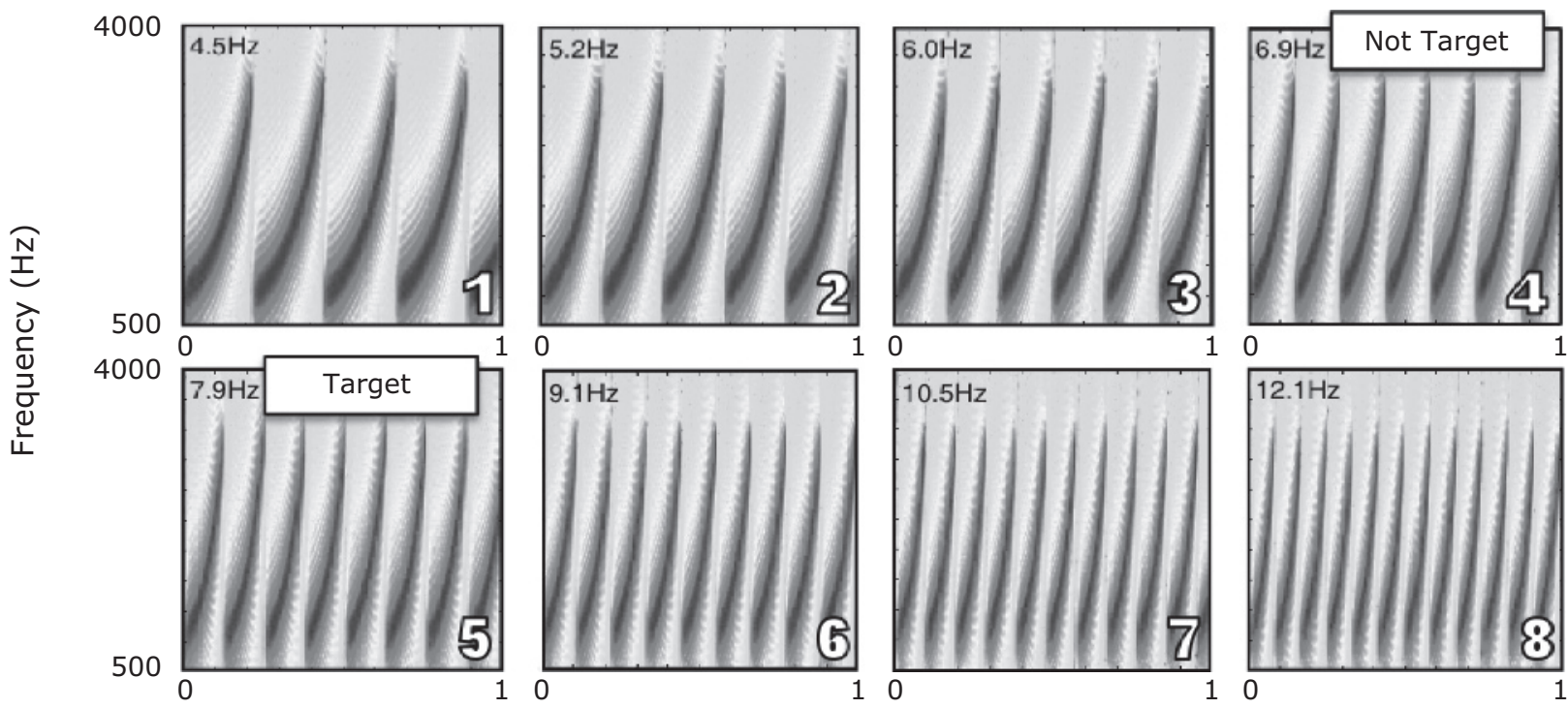

0

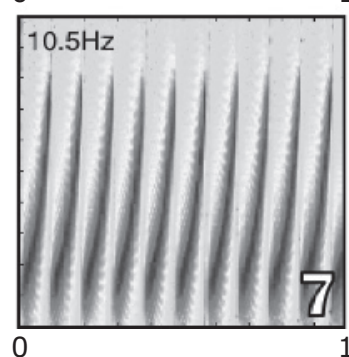

0

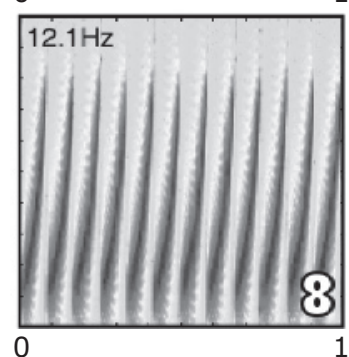

Time (sec)

Figure 1. Spectrograms of the frequency-modulated sweeps used. Stimuli are rank ordered, 1-8, from slowest to fastest. Stimuli used as "Target" and "Not Target" during training are labeled.

key marked "Not Target" if they heard anything else. They were told that during training trials, they would receive feedback but, during testing, they would not. They were also told to guess if they were unsure and that, during the test, there would be many more nontargets than targets.

During discrimination training Sound $5(7.9 \mathrm{~Hz})$ was the target and Sound $4(6.9 \mathrm{~Hz})$ was the nontarget (see Figure 1). A pretraining period served to familiarize participants with their task and the target sound. The pretraining consisted of four sample trials in which one slower and one faster nontarget (one $4 \mathrm{~Hz}$, one $13 \mathrm{~Hz}$ ) and two target sounds were presented. Statements appeared on the screen during these trials that said, "This is not a target" in the presence of the slower and faster sounds and "This is a target" in the presence of the target sound.

Following the pretraining, the participants in each group received their assigned number of training trials, during which they heard Sound 4 and Sound 5 in a pseudorandomized order, so that no more than five of the same sounds were played consecutively. The word "Correct" was presented on the screen if a participant correctly identified Sound 5 as "Target" or Sound 4 as "Not Target." The word "Wrong" was presented if a misidentification was made. After training, the participants were given a reminder on the screen that there would be many more nontargets than targets. They were also reminded that sounds in the test were both slower and faster than the target. All the participants were given 96 trials of generalization testing without feedback. Each sound, 1-8, was presented 12 times in pseudorandomized order so that no more than two of the same sounds were played consecutively.

\section{RESULTS}

Each group's proportion of "Target" responses is plotted in Figure $2 .^{2} \mathrm{~A} 6 \times 8$ ANOVA with training group as the between-participants and stimulus rank as the withinparticipants factor revealed a significant main effect of stimulus rank $\left[F(7,630)=60.97, p<.001, \eta_{\mathrm{p}}^{2}=.404\right]$. This shows that the different stimulus ranks led to different proportions of responding. The main effect of train- ing group was not significant $(F<2)$. The ANOVA also revealed a significant stimulus rank $\times$ training group interaction $\left[F(35,630)=2.316, p=.01, \eta_{\mathrm{p}}^{2}=.114\right]$, indicating differences between the generalization gradients of the groups.

To determine whether there were differences in peak shift between training groups, both the mean and the peak locations of gradients for target responses were calculated for each participant. Gradient means have been used extensively to measure peak shift in past studies (e.g., Thomas et al., 1991). Adding gradient peak allowed a comparison of shift between groups based on actual peak, rather than on peak estimated from gradient means. A peak of a gradient was defined as the stimulus for which maximal responding was observed. If maximum responding was equal for two or more stimuli, the peak was considered to be midway between the stimuli. For example, if Sounds 5 and 6 received the same proportion of responses, the peak was considered to be 5.5. The average gradient mean and gradient peak for each training group are shown in Figure 3.

A one-way ANOVA using the gradient means was significant $\left[F(5,90)=2.77, p=.022, \eta_{\mathrm{p}}^{2}=.13\right]$, with a quadratic contrast $\left[F(1,90)=4.34, p=.04, \eta_{\mathrm{p}}^{2}=.046\right]$. The linear contrast was not significant $[F(1,90)=2.26, p=$ .137]. This shows that gradient means initially increased as training increased, then decreased with further training. One-tailed $t$ tests were performed to determine whether any of the means differed significantly from the target. The $t$ tests were one-tailed because peak shift occurs away from the nontarget training stimulus. All $t$ tests were interpreted with Bonferroni alpha adjustments for 12 comparisons. The $t$ tests revealed that Group 180's gradient mean of 5.76 was significantly different from $5[t(15)=4.539$, 

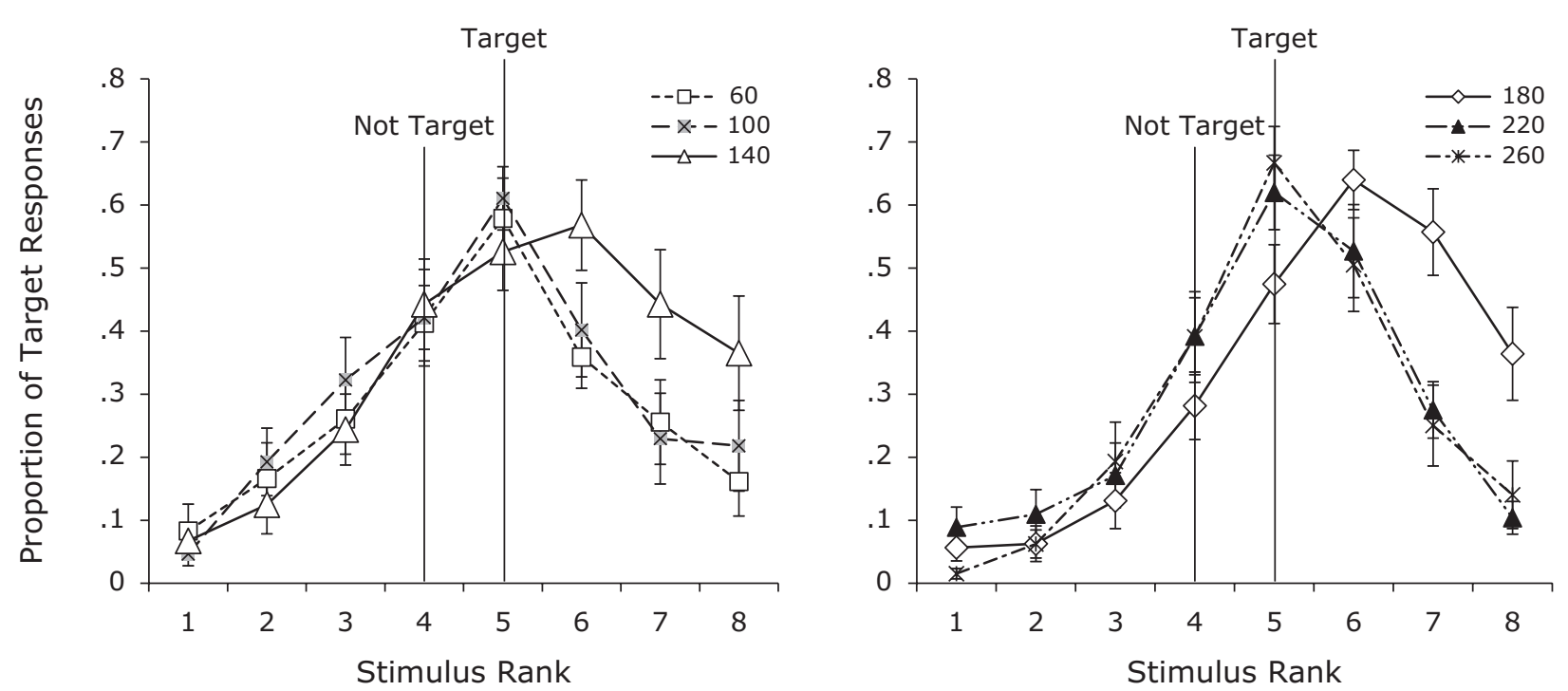

Figure 2. Proportion of "Target" responses for each training group at each level of stimulus rank during the generalization test. "Target" and "Not Target" training stimuli are labeled. Error bars depict the standard errors of the means.

$p<.001$, Cohen's $d=2.34]$. Group 140's gradient mean $[t(15)=1.262, p=.112]$, as well as all other groups' gradient means $(t<1)$, were not. Another one-way ANOVA was performed on the training groups' peaks. This ANOVA was also significant $[F(5,90)=2.531, p=.034$, $\left.\eta_{\mathrm{p}}^{2}=.12\right]$, with a quadratic contrast $[F(1,90)=8.515, p<$ $\left..004, \eta_{\mathrm{p}}^{2}=.086\right]$. The linear contrast was not significant $(F<2)$. One-tailed $t$ tests revealed that the average peak for Group 180 (5.94) was significantly different from 5 $[t(15)=3.137, p=.004$, Cohen's $d=1.62]$. Group 140's average peak of 5.91 was not significantly different from 5 with the Bonferroni correction $[t(15)=2.102, p=.027$, Cohen's $d=1.09$ ]. All the other $t$ tests were also not significant $(t<1)$.
To examine changes in perceptual discrimination ability, $d^{\prime}$ was calculated with correct responses to the target counted as hits, and "Target" responses to Sounds 1-4 counted as false alarms. These values were significantly fit to a linear contrast $\left[F(1,90)=4.851, p=.03, \eta_{\mathrm{p}}^{2}=.051\right]$, indicating that with more training trials, people became better at identifying the target from the side of the distribution containing the nontarget training stimulus. A post hoc least significant difference test also showed that the $d^{\prime}$ values for groups trained for $60(p=.028, S E=.34), 100(p=.041$, $S E=.34)$, and $140(p=.009, S E=.34)$ trials were significantly lower than for participants trained for 260 trials, suggesting that practice led to greater perceptual learning. Figure 4 displays the $d^{\prime}$ values for each training group.

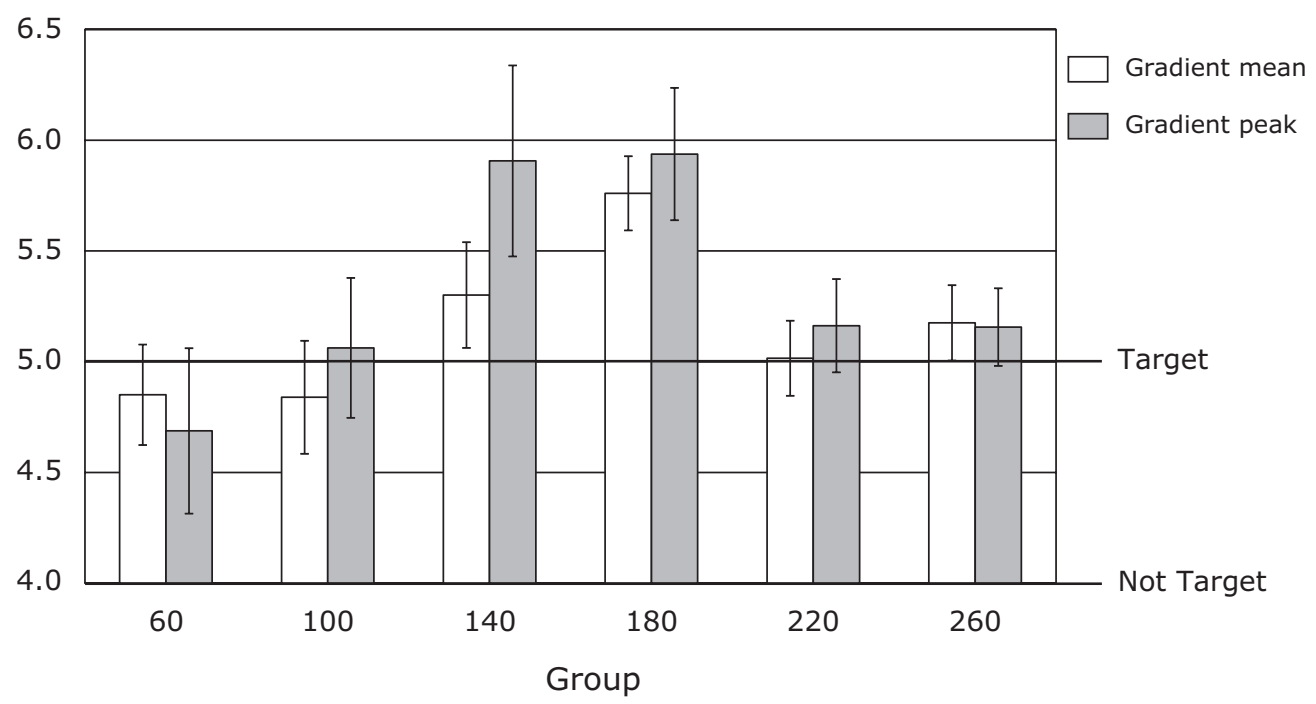

Figure 3. Gradient mean and peak for each training group. The values for the "Target" and "Not Target" sounds are shown. Error bars depict the standard errors of the means. 


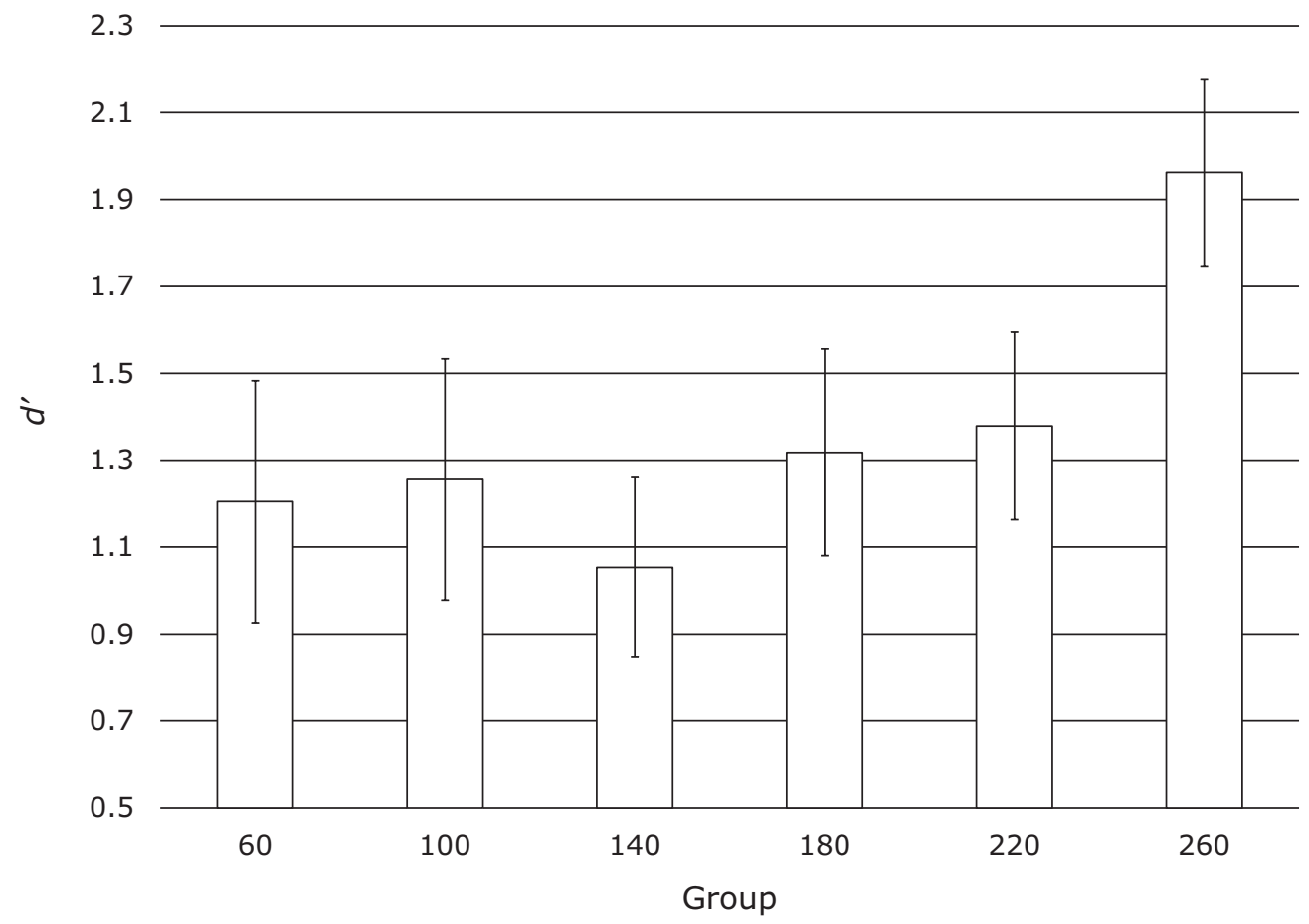

Figure 4. $d$ ' values for each of the training groups. $d$ 's were calculated by counting "Target" responses to the target as hits and "Target" responses to Stimuli 1-4 as false alarms. Error bars depict the standard errors of the means.

\section{DISCUSSION}

In this study, participants who learned to distinguish a fast FM sweep rate (target) from a slightly slower rate (nontarget) with different amounts of training were tested on their ability to identify the target rate among a range of rates. Greater misidentification of a faster novel rate as the target (peak shift) was seen only after intermediate levels of training and dissipated with increased experience. Previous studies have shown that peak shift is stable over time (Spence, 1937; Wisniewski et al., 2009), suggesting that the observed shifts were due to different amounts of training, and not simply to differences between groups in the absolute passage of time.

Current incremental learning theories that rely on static representations do not predict that peak shift should dissipate with extended training. If incremental weight changes between outputs and stable components of a representation caused shift and those weight changes became stronger with practice, the misidentification of a faster stimulus as the target should have become stronger (or at least stable) after it initially appeared. The fact that this did not occur does not mean that peak shift and perceptual learning do not involve any incremental mechanisms, but it does suggest that additional mechanisms are needed to fully explain the data. If it is assumed that other mechanisms of learning dynamically adjust the representational elements evoked by a stimulus over the course of training (Ahissar et al., 2009; Goldstone, 1998; Johansen \& Palmeri, 2002; Mercado, 2008; Nosofsky, 1986; Polley et al., 2006; Smith \& Minda, 1998), an incremental mechanism could predict the results. For instance, if neural representations evoked by the stimuli differentiated or if attention increased the resolution of relevant dimensions, there may have been a point in learning when novel sounds no longer activated the elements of the representation that caused shift. It could also be that switching to a completely new set of elements by switching the area of perceptual processing (Ahissar et al., 2009) nullified the effect of earlier associations between features and outputs that contributed to peak shift.

Why then does peak shift occur if it is ultimately not critical for improved discrimination? One possibility is that the increased generalization to novel stimuli helps to further develop representational precision between stimuli on the dimension. Peak shift has occurred along with increased discrimination performance (Wisniewski et al., 2009), and incremental reweighting theories specifically predict that greater perceptual discriminability accompanies the phenomenon. The present results further suggest that after peak shift dissipates, discrimination performance continues to improve. It could be that the changes underlying peak shift make it easier to refine the representation of the target and that this refinement leads to greater perceptual precision (Blake et al., 2006; Blake et al., 2002). For instance, peak shift may allow more effective engagement of the neural circuits needed for processing the most relevant components (McLaren \& Mackintosh, 2002), allowing differentiation of sensory representations or the allocation of attention to particularly important dimensions. The changes underlying peak shift may similarly make it easier to reconfigure perceptual processing to the appropriate neural circuits (Ahissar 
et al., 2009). Therefore, learning the distinctive elements of a sensory event may make it easier to process its most informative features.

The present results show that peak shift is not always an end state of learning but, rather, can be a transitional state occurring at intermediate levels of training. This is the first report of such transitions in generalization gradients with humans, making it difficult to assess whether similar shifts might occur during learning with other stimulus dimensions or tasks. The results do suggest, however, that learning involves more than just the reweighting of associations between outputs and elements of a static representation and that prior explanations for why peak shift occurs need to be reconsidered.

\section{AUTHOR NOTE}

This work was supported in part by NSF Grant SBE 0542013 to the Temporal Dynamics of Learning Center, an NSF Science of Learning Center. We thank Rachel Tan and Estella Liu for help with data collection and design, and Daniel Schacter, Victor Ferreira, J. Paul Minda, and the reviewers for helpful suggestions. Correspondence concerning this article should be addressed to M. G. Wisniewski, Department of Psychology, University at Buffalo, State University of New York, Park Hall, Buffalo, NY 14260 (e-mail: mgw@buffalo.edu).

Note-Accepted by Cathleen M. Moore's editorial team.

\section{REFERENCES}

Ahissar, M., Nahum, M., Nelken, I., \& Hochstein, S. (2009). Reverse hierarchies and sensory learning. Philosophical Transactions of the Royal Society B, 364, 285-299.

Ashworth, A. R. S., III, \& Dror, I. E. (2000). Object identification as a function of discriminability and learning presentations: The effects of stimulus similarity and canonical frame alignment on aircraft identification. Journal of Experimental Psychology: Applied, 6, 148-157.

BARON, A. (1973). Postdiscrimination gradients of human subjects on a tone continuum. Journal of Experimental Psychology, 101, 337-342.

Blake, D. T., Heiser, M. A., Caywood, M., \& Merzenich, M. M. (2006). Experience-dependent auditory cortical plasticity requires cognitive association between sensation and reward. Neuron, 52, 371381.

Blake, D. T., Strata, F., Churchland, A. K., \& Merzenich, M. M. (2002). Neural correlates of instrumental learning in primary auditory cortex. Proceedings of the National Academy of Sciences, 99, 10114-10119.

BLough, D. S. (1975). Steady state data and a quantitative model of operant generalization and discrimination. Journal of Experimental Psychology: Animal Behavior Processes, 1, 3-21.

DukHAYYIL, A. A., \& Lyons, J. E. (1973). The effect of two types of baseline training on behavioral contrast and the peak shift. Bulletin of the Psychonomic Society, 2, 407-409.

Forster, K. I., \& Forster, J. C. (2003). DMDX: A Windows display program with millisecond accuracy. Behavior Research Methods, Instruments, \& Computers, 35, 116-124.

GaLizIO, M. (1980). Conditional gradient displacements: The effects of conditional discrimination training on human auditory frequency generalization. Journal of Experimental Psychology: Human Learning \& Memory, 6, 766-777.

Gibson, E. J. (1969). Principles of perceptual learning and development. New York: Meredith.

Goldstone, R. L. (1998). Perceptual learning. Annual Review of Psychology, 49, 585-612.

Guillett, L. M., Farrell, T. M., Hoeschele, M., Nickerson, C. M., Dawson, M. R. W., \& Sturdy, C. B. (2010). Mechanisms of call note-type perception in black-capped chickadees (Poecile atricapillus): Peak shift in a note-type continuum. Journal of Comparative Psychology, 124, 109-115.

HEARST, E. (1971). Contrast and stimulus generalization following pro- longed discrimination training. Journal of the Experimental Analysis of Behavior, 15, 355-363.

Johansen, M. K., \& Palmeri, T. J. (2002). Are there representational shifts during category learning? Cognitive Psychology, 45, 482-553.

JUSCZYK, P., \& LUCE, P. (2002). Speech perception and spoken word recognition past and present. Ear \& Hearing, 23, 2-40.

Livesey, E. J., \& McLaren, I. P. L. (2009). Discrimination and generalization along a simple dimension: Peak shift and rule-governed responding. Journal of Experimental Psychology: Animal Behavior Processes, 35, 554-565.

Lynn, S. K., Cnaani, J., \& PapaJ, D. R. (2005). Peak shift discrimination learning as a mechanism of signal evolution. Evolution, 59, 1300-1305.

Martindale, C. (2006). Peak shift, behavioral contrast, and phonological evolution with explanations of the great English vowel shift and Grimm's law. Journal of Cultural \& Evolutionary Psychology, 4, 5-26.

McLaren, I. P. L., \& Mackintosh, N. J. (2002). Associative learning and elemental representation: II. Generalization and discrimination. Animal Learning \& Behavior, 30, 177-200.

Mercado, E., III (2008). Neural and cognitive plasticity: From maps to minds. Psychological Bulletin, 134, 109-137.

Nosofsky, R. M. (1986). Attention, similarity, and the identificationcategorization relationship. Journal of Experimental Psychology: General, 115, 39-57.

Petrov, A. A., Dosher, B. A., \& Lu, Z. (2005). The dynamics of perceptual learning: An incremental reweighting model. Psychological Review, 112, 715-743.

Polley, D. B., Steinberg, E. E., \& Merzenich, M. M. (2006). Perceptual learning directs auditory cortical map reorganization through top-down influences. Journal of Neuroscience, 26, 4970-4982.

Purtle, R. B. (1973). Peak shift: A review. Psychological Bulletin, 80, 408-421.

Queller, S., Schell, T., \& Mason, W. (2006). A novel view of between-categories contrast and within-category similarity. Journal of Personality \& Social Psychology, 91, 406-422.

SAKSIDA, L. M. (1999). Effects of similarity and experience on discrimination learning: A nonassociative connectionist model of perceptual learning. Journal of Experimental Psychology: Animal Behavior Processes, 25, 308-323.

SHEPARD, R. N. (1987). Toward a universal law of generalization for psychological science. Science, 237, 1317-1323.

Smith, J. D., \& Minda, J. P. (1998). Prototypes in the mist: The early epochs of category learning. Journal of Experimental Psychology: Learning, Memory, \& Cognition, 24, 1411-1436.

Spence, K. W. (1937). The differential response in animals to stimuli varying within a single dimension. Psychological Review, 44, 430444.

Spetch, M. L., Cheng, K., \& Clifford, C. W. G. (2004). Peak shift but not range effects in recognition of faces. Learning \& Motivation, 35, 221-241.

Terrace, H. S. (1966). Behavioral contrast and peak shift: Effects of extended discrimination training. Journal of the Experimental Analysis of Behavior, 9, 613-617.

Thomas, D. R., Mood, K., Morrison, S., \& WiertelaK, E. (1991). Peak shift revisited: A test of alternative interpretations. Journal of Experimental Psychology: Animal Behavior Processes, 17, 130-140.

Wills, S., \& Mackintosh, N. J. (1998). Peak shift on an artificial dimension. Quarterly Journal of Experimental Psychology, 51B, 1-31.

Wisniewski, M. G., Church, B. A., \& Mercado, E., III (2009). Learning-related shifts in generalization gradients for complex sounds. Learning \& Behavior, 37, 325-335.

\section{NOTES}

1. For reviews of other theories of peak shift, see Thomas et al. (1991) and Lynn et al. (2005).

2. The percent correct was $28 \%$, averaged across groups, during generalization testing. Chance was $12.5 \%$.

(Manuscript received February 26, 2010; revision accepted for publication June 11, 2010.) 